\title{
Responses of Suprachiasmatic Nucleus Neurons to Light and Dark Adaptation: Relative Contributions of Melanopsin and Rod-Cone Inputs
}

\author{
Elise Drouyer, ${ }^{1,2,3 \star}$ Camille Rieux, ${ }^{1,2,3 \star}$ Roelof A. Hut, ${ }^{1,2,3}$ and Howard M. Cooper ${ }^{1,2,3}$ \\ ${ }^{1}$ INSERM, U846, F-69500 Bron, France, ${ }^{2}$ Department of Chronobiology, Stem Cell and Brain Research Institute, F-69500 Bron, France, and ${ }^{3}$ University of \\ Lyon, Lyon I, F-69000 Lyon, France
}

\begin{abstract}
The circadian oscillator in the suprachiasmatic nucleus $(\mathrm{SCN})$ is entrained to the environmental light/dark cycle through photic information conveyed from the retina. The vast majority of projections to the SCN arise from melanopsin-expressing ganglion cells that are intrinsically light sensitive and that receive inputs from both rods and cones. To investigate the relative contributions of the different photoreceptive systems in shaping the photic signal influencing the circadian clock, we analyzed neuronal responses of single SCN neurons using extracellular electrophysiological recordings under different conditions of light adaptation. In the majority of neurons (78\%), the spike rate is increased by light stimulation whereas the remainder are light-inhibited. The neuronal response to light is composed of several components distinguished by their temporal dynamics and degree of alteration after previous light exposure. SCN neurons display a sustained response to light followed by persistence of the response after light offset. These responses are sluggish and relatively unaffected by previous light exposures. Neurons also respond with a brisk, excitatory ON response and often an OFF response that is either excitatory or inhibitory. $\mathrm{ON}-\mathrm{OFF}$ responses are transient and strongly reduced by previous bright white light exposure. Furthermore, two types of neuronal response patterns can be distinguished by the presence or absence of a slow-transient component that follows the transient $\mathrm{ON}$ response. The transient $\mathrm{ON}-\mathrm{OFF}$ components express light adaptation properties characteristic of retinal channels involving cones, whereas the sustained and persistent components are consistent with in vitro response properties reported for melanopsin-expressing ganglion cells.
\end{abstract}

Key words: retina; circadian; entrainment; phase shift; vision; electrophysiology

\section{Introduction}

In mammals, the suprachiasmatic nucleus (SCN) of hypothalamus contains an endogenous circadian pacemaker that controls most physiological and behavioral rhythms. Entrainment of circadian rhythms and other nonvisual responses to light involve photoreceptors located in both the inner and outer layers of the retina (Hattar et al., 2003; Panda et al., 2003). The main projection to the SCN arises from a subpopulation of intrinsically photosensitive retinal ganglion cells (ipRGCs) (Berson et al., 2002; Warren et al., 2003; Dacey et al., 2005) that also receive synaptic inputs from rods and cones via amacrine and cone bipolar cell synapses (Belenky et al., 2003; Sollars et al., 2003).

Although the absence of all three classes of photoreceptors abolishes circadian entrainment (Hattar et al., 2003; Panda et al.,

Received March 28, 2007; revised June 25, 2007; accepted July 20, 2007.

This work was supported by grants from 6th European Union Framework Grant EUCLOCK, ACT Vieillissement, ACI Neurosciences, Emergence-Rhône-Alpes, and Gis Longévité, and a Fondation Recherche Médicale stipend (E.D.).

${ }^{*}$ E.D. and C.R. contributed equally to this work

Correspondence should be addressed to Dr. Howard M. Cooper, INSERM U846, Stem Cell and Brain Research Institute, Department of Chronobiology, 18 Avenue du Doyen Lépine, F-69500 Bron, France. E-mail: cooper@lyon.inserm.fr.

R. A. Hut's present address: Biological Center, University of Groningen, Kerklaan 30, P.0. Box 14, 9750 AA Haren, The Netherlands.

DOI:10.1523/JNEUROSCI.1391-07.2007

Copyright $\odot 2007$ Society for Neuroscience $\quad$ 0270-6474/07/279623-09\$15.00/0
2003), their relative contributions in photic responses remain unclear. Studies in retinally deficient mice lacking one or more functional photoreceptors show that alteration of behavioral responses to light depends on the photoreceptor deletion and the type of light exposure. Mice lacking melanopsin (Panda et al., 2002; Hattar et al., 2003; Panda et al., 2003), cones, or both rods and cones (Freedman et al., 1999; Semo et al., 2003; DkhissiBenyahya et al., 2007) entrain normally to a bright white light/ dark cycle, but exhibit diminished phase shifts to monochromatic stimulations depending on the wavelength and duration of the light pulse. Mice that predominantly lack rods (retinal degeneration; retinal degeneration slow) have normal phase shifts but fail to entrain at low ( $<1$ lux) light levels (Ebihara and Tsuji, 1980; Mrosovsky, 2003)

These different behavioral consequences could be interpreted to reflect a simple complementarity, with the functional loss of one photopigment compensated by the remaining photopigment(s). Although this view is consistent with the coding of different light stimulus attributes by each photoreceptor type (Dacey et al., 2005; Dkhissi-Benyahya et al., 2007), previous in vitro recordings of ipRGCs reveal a greater range of complexity. The melanopsin driven response to light onset is typically sluggish, persists after light offset and is relatively resistant to bleaching (Berson et al., 2002; Warren et al., 2003; Dacey et al., 2005; 
Wong et al., 2005). This contrasts with phasic cone-driven $\mathrm{ON}-\mathrm{OFF}$ responses present in ipRGCs, consisting of transient increases or decreases in activity that are highly reduced by light bleaching exposures (Dacey et al., 2005). In addition, a surprising degree of heterogeneity within the melanopsin-driven neuronal responses has been demonstrated previously by $\mathrm{Tu}$ et al. (2005).

A potentially diverse range of stimulus responses emanating from different photoreceptor streams thus converges to shape the profile of photic information impinging on individual pacemaker neurons in the SCN. Although photoreceptordeficient animals provide invaluable tools for the study of photoreceptor inputs, genetic alterations are not devoid of developmental consequences (for review, see Gingrich and Hen, 2000) that can range from subtle remodeling to major reorganization of the retina (Marc and Jones, 2003). As specific examples, the absence of one photoreceptor can alter expression of the conserved photopigments (Sakamoto et al., 2004; Barnard et al., 2006; Dkhissi-Benyahya et al., 2007) or of ganglion-cell responses to light ( $\mathrm{Pu}$ et al., 2006). Information from intact animals endowed with a complete set of retinal photoreceptors is thus necessary to fully understand photoreceptor contributions and interactions.

\section{Materials and Methods}

Animals. Male Wistar rats ( $n=18 ; 4-6$ months) from Charles River Laboratories (Charbonnières, France) were maintained on a $12 \mathrm{~h}$ light/ dark cycle. Food and water were available ad libitum and all experiments were in accordance with current international regulations on animal care.

Electrophysiology. Animals were anesthetized with an intraperitoneal injection of $25 \%$ urethane ( $1.2 \mathrm{~g} / \mathrm{kg}$ body weight). Heart rate and temperature were continuously recorded to monitor a stable level of anesthesia. Animals were mounted in a stereotaxic instrument and a dorsal craniotomy was made to allow placement of microelectrodes into the suprachiasmatic region of the hypothalamus. Stereotaxic coordinates of the SCN were determined as $73 \mu \mathrm{m}$ anterior to bregma, $936 \mu \mathrm{m}$ ventral to the cortical surface, and $100 \mu \mathrm{m}$ lateral to the midline. The eyelids were retracted and pupils were pharmacologically dilated by application of $1 \%$ atropine sulfate to the cornea. Eyes and brain surface were maintained hydrated using $0.9 \%$ saline. Extracellular single-neuron activity was recorded using glass, insulated, tungsten microelectrodes (Ainsworth, Wilford, Northamptshire, UK). The exposed tips of the electrode (10 $\mu \mathrm{m}$ length, $1 \mu \mathrm{m}$ at tip) were platinum-gold plated with an impedance of $0.5-1 \mathrm{M} \Omega$ to effectively isolate single neurons. Recordings and stimulations were made using a Tucker-Davis Technologies (Alachua, FL) digital recording system. A multispike waveform discriminator (Alpha Omega, Nazareth, Israel) was used to track on-line the activity of individual neurons based on spike amplitude and shape.

Light-stimulation protocols. Animals were exposed to a $500 \mathrm{~nm}$ monochromatic stimulations. Stimulation profiles and irradiance were controlled by a computer and monochromatic light was produced using a tungsten halogen light source, infrared absorbing filters, collimating lenses, and a Schott interference filter $(10 \mathrm{~nm}$ band width at half peak transmission). Light was projected through an opal diffuser into a stimulation sphere centered with reference to the animals head, thereby providing a uniform illumination that encompassed the entire visual field. Using an irradiance of $2 \times 10^{14}$ photons $/ \mathrm{cm}^{2} / \mathrm{s}$, neurons were recorded under two conditions: dark adaptation (DA) and light adaptation (LA) (Fig. 1). DA consisted of 30 min of total darkness (allowing the rods and cones to recover full sensitivity) (Fain et al., 2001) preceding the monochromatic test stimulation, whereas in the LA condition, the monochromatic stimulation is preceded by a bright white light stimulation (56 $\mathrm{W} / \mathrm{m}^{2} / \mathrm{s}$ at $400-700 \mathrm{~nm}\left(>10^{17}\right.$ photons $/ \mathrm{cm}^{2} / \mathrm{s} ; 3000$ photopic lux) lasting $1 \mathrm{~min}$ and ending $1 \mathrm{~min}$ before the test stimulation, during which the animal is exposed to complete darkness to allow the neurons to return to a stable baseline.

Light adaptation is a classical strategy used to study photoreceptive channels, from single photoreceptors to higher visual processes, by selectively reduce specific response components (Knoblauch and Shevell, 2001; Dacey et al., 2005). Light adaptation involves mechanisms of both chromophore depletion and a neural process (producing a maintained decrease of intracellular $\mathrm{Ca}^{2+}$ concentration affecting the transduction cascade) leading to desensitization of the photoreceptor (for review see, Fain et al., 2001). Although ipRGCs, like rods and cones, have been shown to adapt to light, they appear more highly resistant to light bleaching (Wong et al., 2005). Recordings in intact retina indicate that after high light exposures, ipRGCs continue to respond to light whereas nonmelanopsin-expressing cells fail to respond, presumably resulting from extensively bleaching of rods and cones (Berson et al., 2002; Warren et al., 2003). This desensitizing effect is analogous to the use of synaptic blockers to prevent rod and cone synaptic input to ipRGCs used during in vitro retinal studies. An advantage of this approach is that by alternating the light adaptation exposures with appropriate dark adaptation recovery periods, repeated evoked responses can be assayed from the same neuron.

Stimulations were repeated three times for each condition and for each neuron. A full protocol lasted $2 \mathrm{~h}$. Five neurons could be recorded for longer periods (up to $5 \mathrm{~h}$ ) and were further investigated at lower irradiances under the DA condition. Stimulations were repeated three times at $2 \times 10^{13}$ photons $/ \mathrm{cm}^{2} / \mathrm{s}$ and two times at $2 \times 10^{12}$ photons $/ \mathrm{cm}^{2} / \mathrm{s}$ and $2 \times 10^{11}$ photons $/ \mathrm{cm}^{2} / \mathrm{s}$ for each neuron.

Selection of light-responsive neurons. The following criteria were used to select light-responsive neurons. Once the neuronal signal was isolated, the capacity to respond to light was tested using a stimulation protocol consisting of three successive monochromatic light pulses alternating with darkness lasting $10 \mathrm{~s}$ each at an irradiance of $2 \times 10^{14}$ photons/ $\mathrm{cm}^{2} / \mathrm{s}$. Neurons that displayed a detectable alteration in firing rate during the light pulses were classified as light responsive and retained for analysis. Neurons that expressed a baseline firing rate but were unresponsive to light stimulation, or neurons that were unable to maintain a clear response to light, were not conserved for additional study. Some neurons that were initially classified as light responsive subsequently decreased or ceased light responsiveness, or became unstable or erratic in their responses. This prevented the recording of a complete data set though the duration of the three response cycles. These neurons were thus excluded from the analysis.

Of a total of 287 neurons encountered during the descents, 132 neu- 

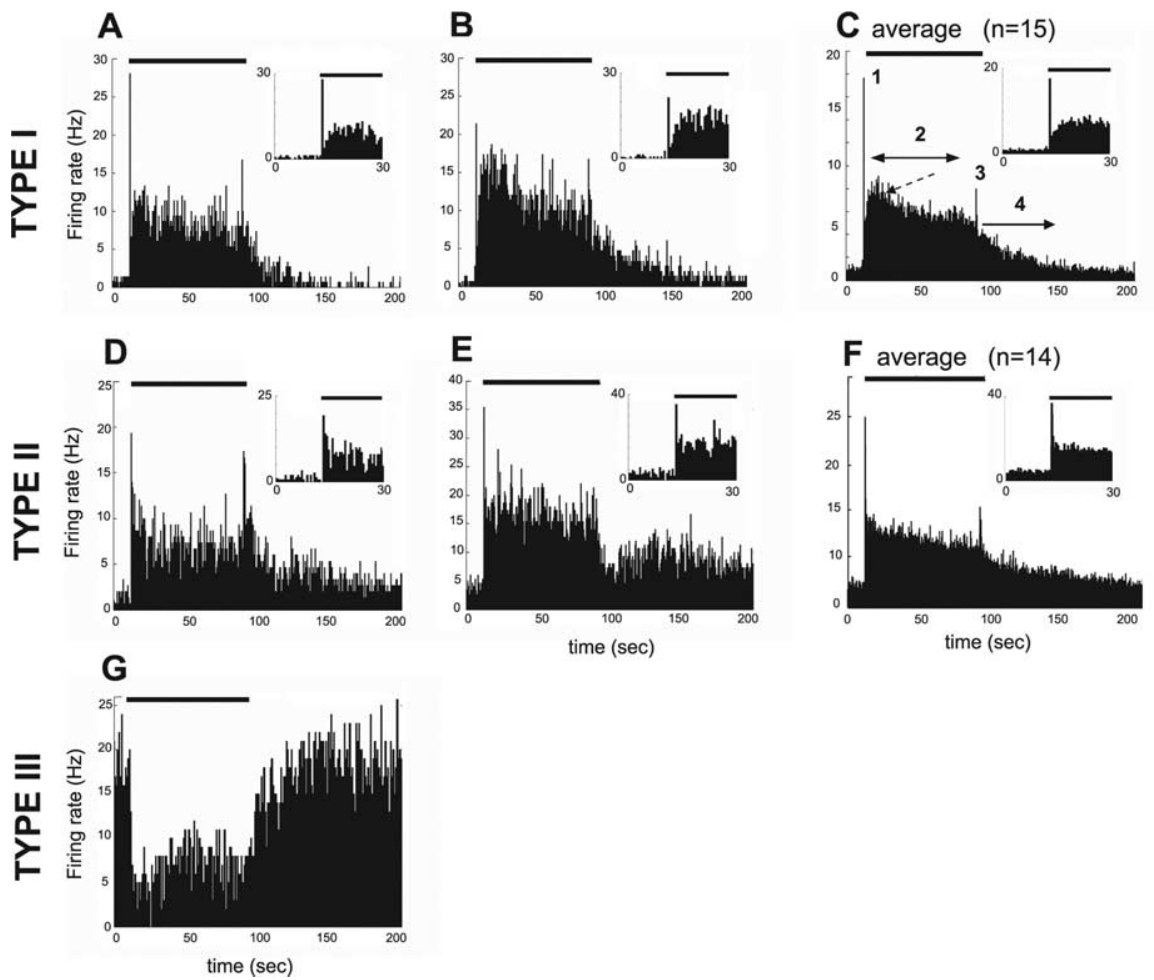

Figure 2. Single-unit response of SCN neurons to light in the DA condition. After $30 \mathrm{~min}$ of dark adaptation, the animal is exposed to a 90 s duration pulse (black bar) of monochromatic light at $500 \mathrm{~nm}\left(2 \times 10^{14} \mathrm{photons} / \mathrm{cm}^{2} / \mathrm{s}\right)$. The inset in each panel depicts the first $30 \mathrm{~s}$ of the recording (bin size, $0.1 \mathrm{~s}$ ). $A-G$, Three types of light responses were found: light-excited neurons with a slow-transient component (type $\mathbf{F} \boldsymbol{A}-\boldsymbol{C}$ ) or without a slow-transient component (type II; $\boldsymbol{D}-\boldsymbol{F}$ ), and light-inhibited neurons (type III, G). The response to light includes several temporally distinct components (shown in C): fast-transient ON response (1), sustained response (2), transient OFF response (3), and poststimulus persistence (4). The slow transient component is indicated by the gray dashed arrow. $\boldsymbol{A}-\boldsymbol{C}$, Individual $(\boldsymbol{A}-\boldsymbol{B})$ and average $(\boldsymbol{C} ; n=15)$ recordings of type I neurons showing the initial transient $0 \mathrm{~N}$ response followed by a slow-transient component that reaches the maximum firing rate within $\sim 10$ s (arrow). $\boldsymbol{D}, \boldsymbol{E}$, Recordings of individual type II neurons that lack the slow transient. Note the presence in these neurons of a transient $0 \mathrm{FF}$ response that is excitatory $(\boldsymbol{D})$ or inhibitory $(\boldsymbol{E}) . \boldsymbol{G}$, Example of a light inhibited neuron, estimated to represent $22 \%$ of the light responsive cells in the SCN.

rons (46\%) were clearly light responsive. A total of 29 of these light responsive neurons could be fully characterized according to the stringent criteria. Recordings from an individual animal generally lasted $18-30 \mathrm{~h}$, usually beginning at Zeitgeber time 4 . We did not measure any significant nycthemeral variations of the basal firing rate of neurons in the dark or in their response amplitude to light stimulation.

Analysis. Recordings of neuronal spike frequencies were analyzed using software developed under Matlab 7.0 (Mathworks, Natick, MA). The bin size used for averaging responses was $0.1 \mathrm{~s}$. For purposes of illustration a bin size of $0.2 \mathrm{~s}$ is used unless otherwise indicated. Parameters measured included the latency of the response, the average firing rate, and the amplitude of the response. Several different response components could be differentiated according to these parameters and their time of occurrence in relation to the light stimulation (see Results).

Statistical analysis was computed using SigmaStat software (Systat Software, Point Richmond, CA). The nonparametric Mann-Whitney test was used for comparisons between groups and the nonparametric Wilcoxon test for repeated measures. The results are expressed as means \pm SEM.

Histology. At the end of the experiment, the final electrode track was marked with 1-3 electrolytic lesions. The animal received a lethal dose of sodium pentobarbital, and the brain removed and fixed with by immersion in Zamboni fixative (4\% phosphate buffered paraformaldehyde, picric acid) during $24 \mathrm{~h}$. Brains were then cryoprotected by immersion in $30 \%$ sucrose in phosphate buffer $(0.01 \mathrm{M})$, pH 7.4, for $24 \mathrm{~h}$. Serial coronal sections were made at $50 \mu \mathrm{m}$ on a freezing microtome. The positions of the recording sites in the SCN were confirmed by comparing the stereo- taxic coordinates of recorded neurons to the positions of the electrode track locations of the marked lesions.

\section{Results}

Response patterns in dark-adapted conditions: evidence for two groups of components with different kinetics

A total of 132 light-responsive neurons were encountered in the SCN, of which the majority of cells $(78 \%)$ were light excitatory and responded to photic stimulation by an increase in their firing rate. Figure $2 A-G$ illustrates several examples of lightresponse patterns of individual SCN neurons after a dark-adaptation period of 30 min. Under these conditions, rods and cones are fully responsive to light during the whole field exposure of both eyes to monochromatic light stimulation. However, at the high irradiance used $(500 \mathrm{~nm}$; $2 \times 10^{14}$ photons $/ \mathrm{cm}^{2} / \mathrm{s}$ ), rods are probably not responsive to the $90 \mathrm{~s}$ light pulse (Aggelopoulos and Meissl, 2000; Nathan et al., 2006). A total of 29 neurons were fully characterized under DA conditions. The average spontaneous firing rate of these SCN neurons in the dark is $1.93 \pm$ $0.56 \mathrm{~Hz}$. Despite a certain degree of diversity among individual SCN neurons, the response to light can be characterized by several temporally defined transient and sustained components (Fig. 2).

The onset of light stimulation leads to a rapid, brisk onset response of short latency $(0.13 \pm 0.01 \mathrm{~s})$ and of high spike frequency $(20.80 \pm 2.03 \mathrm{~Hz})$. This initial fast component is always excitatory, transient, and generally lasts $<500 \mathrm{~ms}$ (Fig. $2 A-F$ ), although in certain neurons the firing rate shows a gradual decrease after the initial transient response (Fig. 2D). This rapid phasic component is referred as fast-transient $\mathrm{ON}$ response.

After the initial fast-transient response, SCN neurons show a tonic sustained response throughout the entire period of light exposure. Quantified as the average firing rate during the entire period of light stimulation (excluding the initial $500 \mathrm{~ms}$ transient), this sustained response equals $8.23 \pm 1.01 \mathrm{~Hz}$ for all neurons $(n=29)$. However, based on the profile of the tonic firing rate discharge pattern two groups of neurons can be distinguished.

Type I neurons ( 15 of 29) display a slow-transient component expressed as a slow initial increase in firing rate after the fast transient $\mathrm{ON}$ response that attains a maximum, on average, within $10.00 \pm 0.46 \mathrm{~s}$ after light onset, and then declines gradually to a stable sustained level. The slow transient is defined by an increase in firing rate $\geq 25 \%$ than the averaged sustained response rate, lasting at least $3 \mathrm{~s}$ and occurring within the first one-third of the light exposure. Figure 2, $A$ and $B$, represents two examples of this type I response pattern, and Figure $2 C$ shows the average response pattern.

Type II neurons lack the slow-transient response (14 of 29) and only express the stable sustained response that sharply increases in firing rate after the initial transient and subsequently 
maintains a stable response rate throughout the duration of light exposure. Figure 2, $D$ and $E$, represents two examples of this type II response pattern and Figure $2 \mathrm{~F}$ shows the average response profile.

Type I and type II neurons also significantly differ in their basal firing rate and in the firing rate of the sustained response to light (type I basal rate, $0.81 \pm 0.19 \mathrm{~Hz}$; type II basal rate, $3.11 \pm$ $1.06 \mathrm{~Hz}$; Mann-Whitney, $p<0.05$ ) whereas both the $\mathrm{ON}$ and sustained responses are similar in neurons type I and II (MannWhitney, $p=0.116$ and $p=0.111$, respectively)

At light offset, approximately one-half of the neurons (13 of 29) display a third component consisting of a fast-transient OFF response. An excitatory OFF response is present in both type I ( 5 of 15) and type II (5 of 14) neurons (Fig. $2 A, B, D$ ), whereas an inhibitory OFF response is less common and only observed in type II neurons (3 of 14) (Fig. 2E). The OFF excitatory response is of short latency $(0.17 \pm 0.02 \mathrm{~s})$, occurs within first $500 \mathrm{~ms}$ after light offset, and is of relatively high amplitude $(16.61 \pm 1.70 \mathrm{~Hz})$. The firing rate of the transient excitatory OFF response is typically less that of the ON transient response. The OFF inhibitory response was difficult to quantify given the small number of neurons and was not analyzed further.

After the end of the light stimulation, neuronal activity slowly returns to baseline in an average time of $44.95 \pm 2.34 \mathrm{~s}$ (Fig. 2). The duration of this poststimulus persistence response is defined as the time required to return to a response level equal to $50 \%$ of the sustained response after light offset. The firing rate of the response is quantified as the averaging firing rate within this duration. On the average, the return to baseline firing rate is similar in type I (46.29 $\pm 2.86 \mathrm{~s}$ ) (Fig. $2 C$ ) and type II neurons (43.27 \pm 3.97 s) (Mann-Whitney test, $p=0.369$ ) (Fig. $2 F$ ).

In the dark-adapted state, all of the recorded neurons display the fast-transient $\mathrm{ON}$, sustained, and poststimulus response components. No pure fast-transient ON or OFF or isolated sustained-only responses were observed.

All of the neurons discussed above show an increase in firing rate in response to light stimulation. However, we estimate that in $22 \%$ of the light-responsive neurons in the SCN, neuronal activity is inhibited by light (Fig. 2G) (type III). The relative instability of light responsiveness in these neurons did not allow a complete characterization over the entire experimental protocol. Most of these light-inhibited neurons either rapidly ceased to respond to light stimulation or showed a progressive decrease in the response after a series of light stimulations. This also occurred in some of the light-excited neurons that, accordingly, were not included in the analysis. In some rare cases, the initial inhibitory response of the neuron to light spontaneously reversed into an excitatory response during a subsequent bout of light stimulation. Despite the difficulty to perform a full recording protocol from lightinhibited neurons, observation of the firing rate-profiles of light-inhibited neurons during the light stimulation tests shows that the response patterns were basically similar to those of light-activated neurons. In general, the responses display a transient suppression of activity at light onset that continues during stimulation, followed by a subsequent persistence of suppression after light offset. Because this inhibitory response pattern has not been observed in ipRGCs, these cells may be postsynaptic targets of SCN neurons that receive direct contacts from retinal fibers because retinal inputs involve the excitatory neurotransmitter glutamate (for review, see Ebling, 1996; Rea, 1998).

\section{Effect of light adaptation on neuronal response pattern:} response components are differentially affected

Of the 29 neurons recorded under the DA condition, 27 (13 of type I and 14 of type II) neurons were also fully characterized after LA. In this condition, the test stimulation was preceded by a full-field bright white light stimulation (preadaptation "bleaching" stimulation) known to induce a prolonged desensitization of the rods and cones (Baylor and Hodgkin, 1974; Fain et al., 2001). Figure 3 shows several examples of individual and average recordings in both the DA and LA conditions for type I and type II neurons. The bright light exposure that precedes the test stimulation obviously affects the response pattern, thereby showing that the immediate previous light history has a profound impact on the photic responses of SCN neurons. All of the neurons show lower average spike frequencies during the light stimulation in the LA condition compared with the DA condition, although the fast-transient $\mathrm{ON}$ and the sustained responses are differentially affected by previous light exposure.

After light adaptation, the fast-transient ON component observed in DA is either totally abolished or, when detectable, is strongly diminished in amplitude for both type I and type II neurons. The average reduction is $67.13 \pm 7.94 \%$ (Figs. 3, 4). The transient excitatory OFF response is similarly affected and, on average, is reduced by $39.49 \pm 5.75 \%$. Only two neurons (type I) retain a detectable OFF response. The reductions in responsiveness of the transient $\mathrm{ON}-\mathrm{OFF}$ components are consistent with and typical of rod- and cone-driven retinal mechanisms (Berson et al., 2002; Dacey et al., 2005).

In contrast, the firing rate of the sustained response is less affected by light adaptation than the transient responses (Fig. 3). On the average, for all of the neurons, the sustained response is decreased by only $21.81 \pm 6.28 \%$ in LA, although the degree of reduction of the response differs between type I and type II neurons. In type I neurons, the firing rate amplitude is significantly reduced for both the slow-transient $(38.73 \pm 8.54 \%)$ and the sustained responses $(31.20 \pm 10.06 \%)$ (Figs. $3 A, 4 A)$. In contrast, type II neurons do not display a significant reduction of the sustained component $(13.64 \pm 7.72 \%)$ after bright light exposure (Figs. $3 B, C, 4 B$ ). Four of the neurons actually showed a slight increase in firing rate (Fig. $3 C$ ).

The firing rate of the poststimulation persistence after light offset also remains unaltered by the previous light exposure in both type I and II neurons (average decrease, $2.82 \pm 7.27 \%$ ) (Fig. 3 ). The greater resistance of the sustained and poststimulation persistence in the LA condition is consistent with the idea that both rely on the same response mechanism. These two response components are similar to the response properties described previously in melanopsin ipRGCs (Berson et al., 2002; Dacey et al., 2005; Tu et al., 2005).

Because the various light-responsive components are differentially reduced by previous light exposure, we used a subtractive method to derive the pattern of response profiles of the components that are most affected by light adaptation. Figure 3, $D$ and $E$ (top), shows the averaged responses for type I and type II neurons in the LA condition (in gray) superimposed on the averaged responses in the DA condition (in black). The difference in shading allows a distinction between the two response patterns and overall consequences of previous light adaptation in both type I and type II neurons. Subtracting the responses of LA from DA unmasks the response amplitudes attenuated or abolished by light adaptation (Fig. 3D,E, bottom). These histograms reveal lightadapted responses that closely resemble cone-mediated responses of melanopsin ganglion cells illustrated by Dacey et al. 


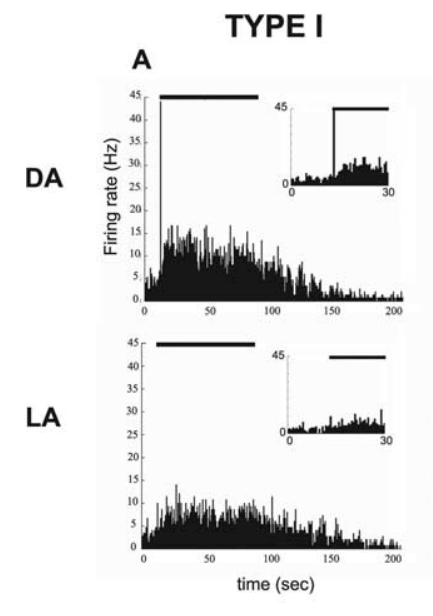

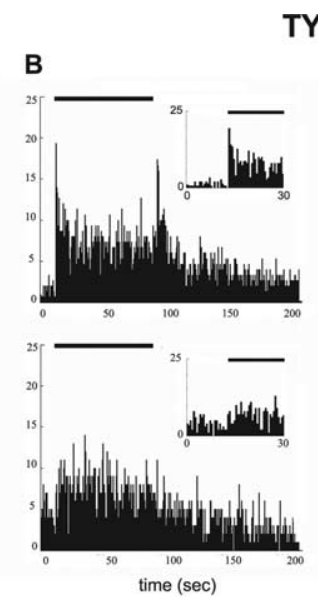

TYPE II
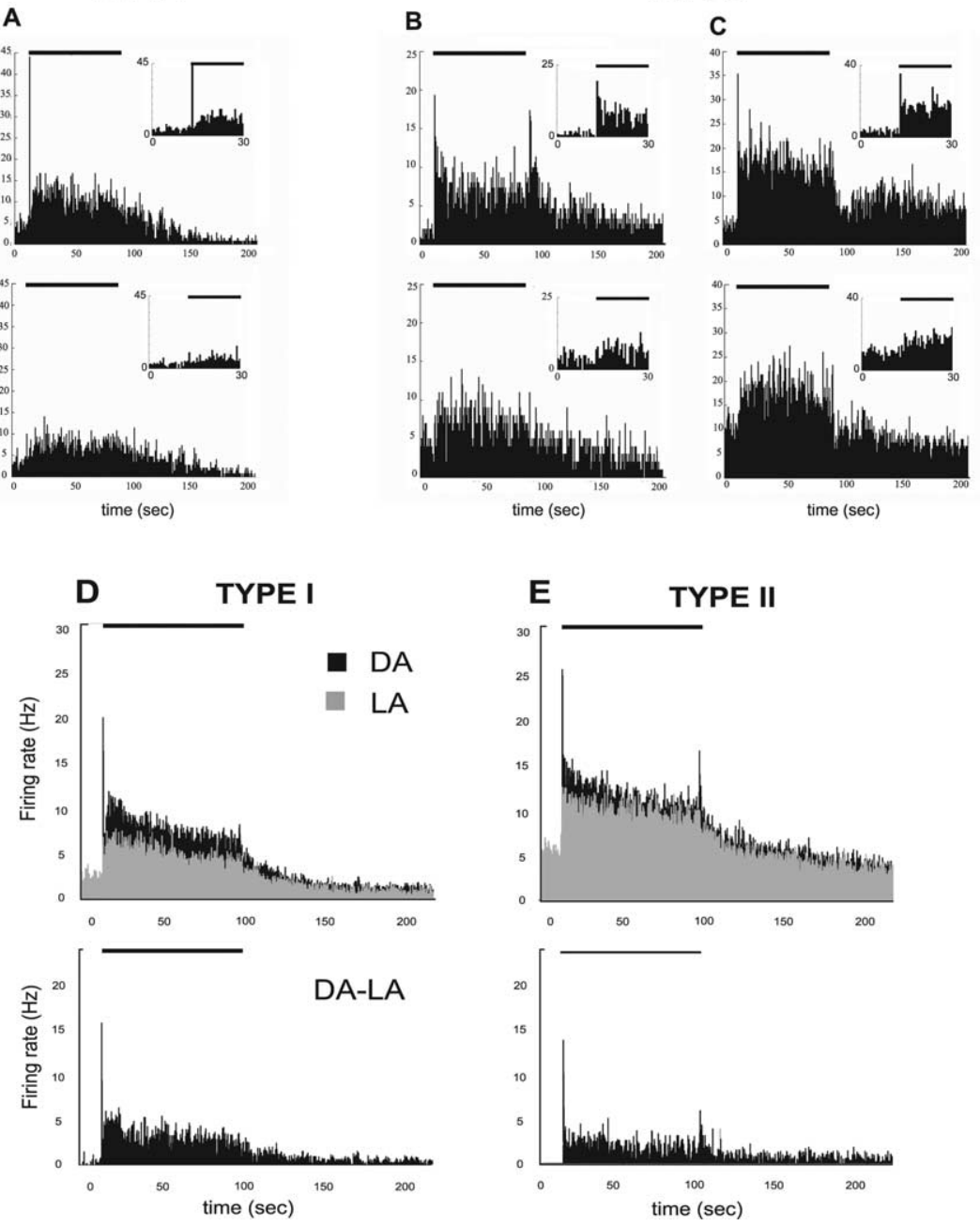
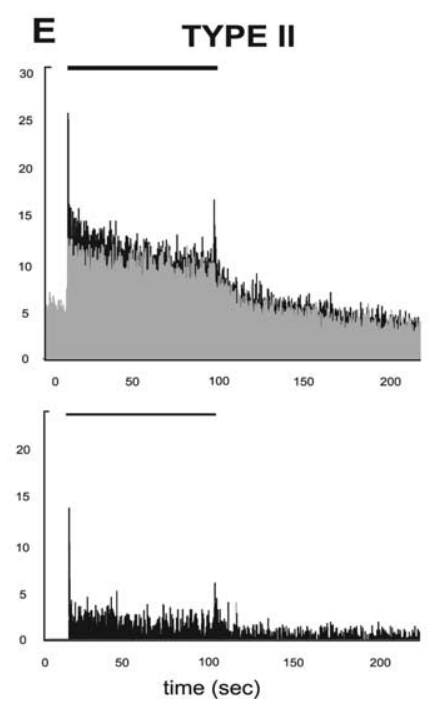

Figure 3. Single-unit light responses of individual SCN neurons in the DA and the LA conditions. For light adaptation, the animal was exposed to $1 \mathrm{~min}$ bright white light $\left(56 \mathrm{~W} / \mathrm{m}^{2} / \mathrm{s}\right)$ followed by $90 \mathrm{~s} \mathrm{stimulation} \mathrm{(black} \mathrm{bar)} \mathrm{of} \mathrm{monochromatic} \mathrm{light} \mathrm{at}$ $500 \mathrm{~nm}\left(2 \times 10^{14}\right.$ photons $\left./ \mathrm{cm}^{2} / \mathrm{s}\right)$. Insets represent the first $30 \mathrm{~s}$ of the recordings (bin size, $\left.0.1 \mathrm{~s}\right) . \boldsymbol{A}$, Example of a type I neuron that exhibits an initial slow-transient component associated with the sustained response in DA. $\boldsymbol{B}, \boldsymbol{C}$, Examples of two type II neurons that do not exhibit an initial slow-transient component in DA. Note that for both types of neurons, light adaptation dramatically affects the fast-transient $\mathrm{ON}$ and $0 \mathrm{FF}$ responses to light. $\boldsymbol{D}, \boldsymbol{E}$, The averaged responses of type I (D) and II (E) neurons are shown the top with average LA response (gray), superimposed on the average DA response (black). The bottom in $\boldsymbol{D}$ and $\boldsymbol{E}$ show the subtracted averaged LA from DA responses to light for both types of neurons, revealing the response pattern of the light-attenuated components. The transient $\mathrm{ON}$ and $\mathrm{OFF}$ responses are clearly visible, indicating that these components are most affected by previous light exposure. This response pattern is typical of rod - cone responses. A part of the sustained response and of the poststimulus persistence of the response is also affected by the light preadaptation.

(2005). These typical light-adaptation properties convincingly suggest that this contribution is derived from cone (and/or rod) photoreceptors.

\section{Effect of irradiance on the response pattern}

Five of the 29 neurons that were recorded in DA at a high irradiance $\left(2 \times 10^{14}\right.$ photons $\left./ \mathrm{cm}^{2} / \mathrm{s}\right)$ were also tested at a lower irradiance $\left(2 \times 10^{13}\right.$ photons $\left./ \mathrm{cm}^{2} / \mathrm{s}\right)$ in DA conditions. The other 24 neurons could not be investigated further after the long DA-LA protocols because either the neuronal response became inconsistent or the electrophysiological signal was lost. Because of the small sample size, statistical analysis of the quantitative alterations was not performed.

An example of an individual type I neuron is shown in Figure $5 A$, and the average responses of the four type I neurons are displayed in Figure $5 B$. Recordings of the single type II neuron are shown in Figure $5 C$. Both types show a similar pattern of reduction in firing rate with the reduction in irradiance and the average change in individual parameters were calculated for the four type I and single type II neurons. A reduction in the irradiance by one log unit within the photopic range leads to a significant overall decrease of the firing rate of the SCN neuronal response (Fig. 5), as would expected from luminance coding units. The average overall reduction in firing rate (all components) during the light stimulation at $2 \times$ $10^{13}$ photons $/ \mathrm{cm}^{2} / \mathrm{s}$ is $47.9 \%$ that of the rate at $2 \times 10^{14}$ photons $/ \mathrm{cm}^{2} / \mathrm{s}$. The firing rate of the fast-transient $\mathrm{ON}$ response is strongly affected by the reduction in irradiance and all five neurons show a substantial decrease in amplitude (67.4 \pm $3.53 \%$ ) (Fig. 5). Three neurons displayed an excitatory OFF response at $2 \times 10^{14}$ photons $/ \mathrm{cm}^{2} / \mathrm{s}$ that was undetectable at the lower irradiance (Fig. 5). One neuron displayed an inhibitory OFF response at $2 \times 10^{14}$ photons $/ \mathrm{cm}^{2} / \mathrm{s}$, but was not detectable at $2 \times 10^{13}$ photons $/ \mathrm{cm}^{2} / \mathrm{s}$.

The slow-transient component (four type I neurons) is totally absent at the lower irradiance of $2 \times 10^{13}$ photons/ $\mathrm{cm} 2 / \mathrm{s}$ (Fig. $5 A, B$ ). The sustained response is decreased in all neurons (average reduction, $27.51 \pm 0.59 \%)$. Four of the five neurons show a slight decrease in the poststimulus persistence (average reduction, $8.53 \pm 0.52 \%)$. Based on response amplitude, both the sustained and the fasttransient components of the light response express properties of irradiance encoding, although the fast-transient ON and OFF components are more susceptible to alteration for the decrease in irradiance.

Examination of the temporal aspects of the response reveals that the latency of the fast-transient ON component is unaffected by the decrease in irradiance (average latency, $0.14 \pm 0.02 \mathrm{~s}$ at $2 \times 10^{13}$ photons $\left./ \mathrm{cm}^{2} / \mathrm{s}\right)$. The only excitatory OFF response detectable at $2 \times 10^{13}$ photons $/ \mathrm{cm}^{2} / \mathrm{s}$ showed no change in latency compared with the higher irradiance $(0.2 \mathrm{~s}$ at both irradiances) (data not shown). In contrast, at 1 log unit lower irradiance, the time required for the sustained component to attain the plateau level increases to $36.78 \pm 2.65 \mathrm{~s}$. Finally, the duration of the response persistence after light offset remains relatively unchanged at lower irradiance and is only reduced by $6.32 \pm 3.44 \%$.

The amplitude and kinetics of the sustained and persistent components compared with the transient components are differentially affected by the modulation of irradiance. This reinforces the idea that these two sets of components rely on different channels with distinct photoreceptive properties.

The responses of these five neurons were also recorded at 1 - to 2-log unit-lower irradiances. At $2 \times 10^{12}$ photons $/ \mathrm{cm}^{2} / \mathrm{s}$, only two of the neurons displayed a clear response to light (Fig. 5D). 

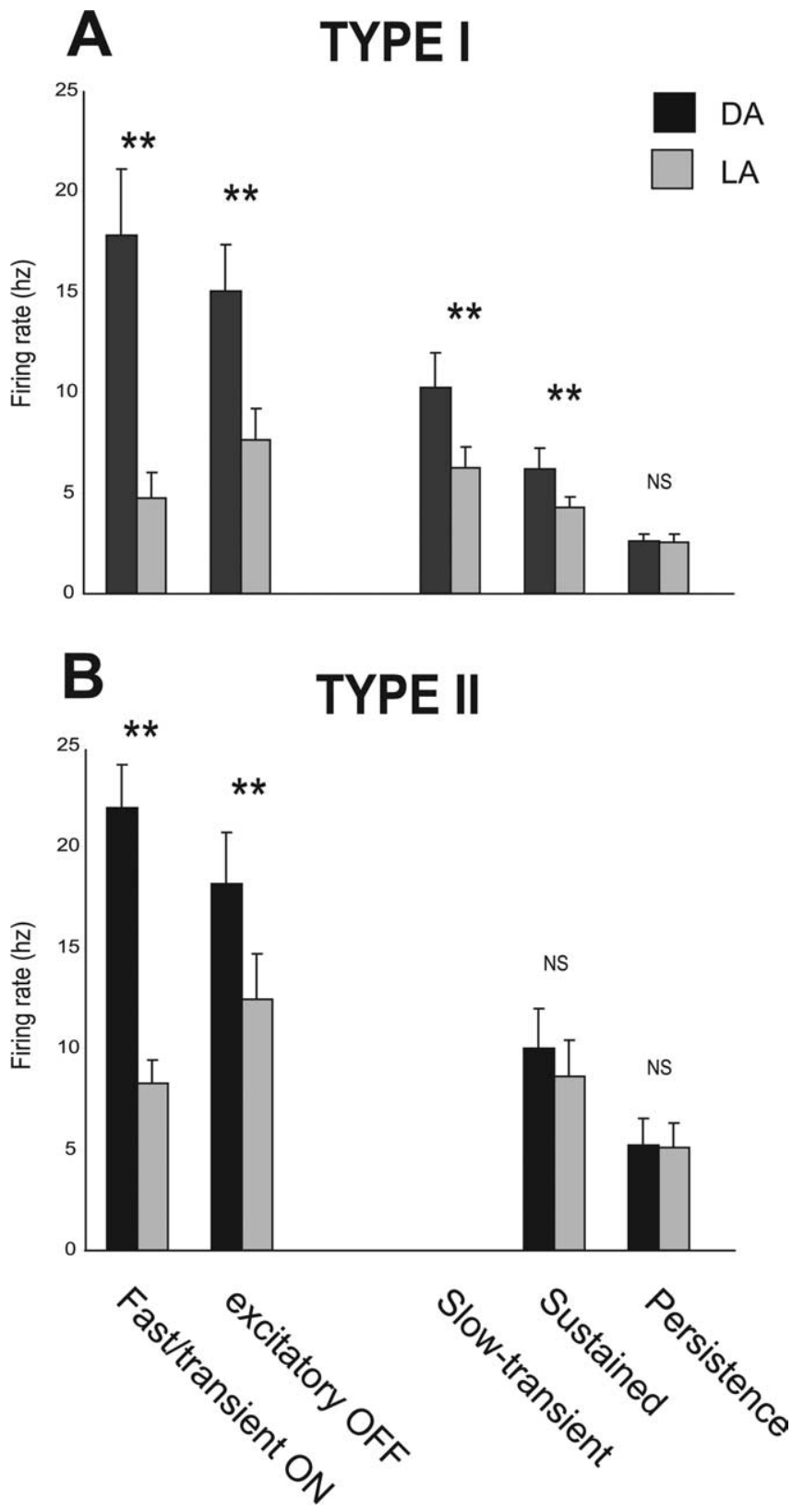

Figure 4. $A, B$, Effect of light adaptation on the firing rate of different components of the response in type $I(\boldsymbol{A})$ and type II $(\boldsymbol{B})$ neurons shown for DA and LA conditions. The transient $0 \mathrm{~N}$ and transient OFF responses are the most affected by light adaptation (OFF responses, $n=10$, pooled for statistical analysis). In type I neurons, both the slow-transient and sustained responses are significantly decreased by light adaptation, whereas in type II neurons the sustained response remains unchanged. The poststimulus persistence of the response is unaffected by previous light adaptation. Recall that because type II neurons do not exhibit a slow-transient component, this value is not shown in the histogram in $\boldsymbol{B}$. Values are expressed as the mean + $\operatorname{SEM}(n=27) .{ }^{* *} p<0.01$ (Wilcoxon test).

Response latency was very high and the firing rate extremely sluggish. At the lowest irradiance tested, $2 \times 10^{11}$ photons $/ \mathrm{cm}^{2} / \mathrm{s}$, we did not observe any detectable neuronal response in the present light-exposure conditions (Fig. 5E).

\section{Discussion}

The SCN neuronal response to light is complex and systematically includes two response components that are distinguished by different temporal kinetics, resistance to bleaching and responses to irradiance. Fast-transient ON (excitatory) and transient OFF (excitatory or inhibitory) responses are of short latency, are strongly influenced by previous light exposure, and rapidly decrease in amplitude at lower irradiance levels. The sustained components, including the slow-transient component and the persistence of the response after light offset, show longer latencies, higher resistance to bleaching, and are less affected by decreases in irradiance. The properties of these two groups of response components are consistent with retinal channels involving outer retinal cone photoreceptors and inner retinal melanopsin ipRGCs that both encode irradiance at photopic levels, but are differentially modulated.

\section{The fast-transient $\mathrm{ON}-\mathrm{OFF}$ components are derived from outer retinal photoreceptors}

Excitatory transient ON responses are observed in all SCN neurons, whereas transient OFF responses (mainly excitatory) are present in one-half of the neuronal population. Both transients are more prominent in the dark-adapted condition and modulated by irradiance. These properties are strikingly different from the sustained responses reported for melanopsin ganglion cells, but are fully consistent with the response properties of retinal channels involving cones and rods (Volgyi et al., 2004; Wassle, 2004). However, although both cones and rods display transient ON-OFF responses to light, it is unlikely that rods contribute significantly to the responses recorded in the present study. Rod transients have longer latencies $(>200 \mathrm{~ms}$ ) and the intensities used in our study correspond to the cone-dominated photopic domain (Aggelopoulos and Meissl, 2000; Nathan et al., 2006) that precludes rod influences at the cone-bipolar interface (Bloomfield and Dacheux, 2001). It is nevertheless premature to discount a potential contribution from rods at lower light levels. At scotopic levels, Aggelopoulos and Meissl (2000) report a roddominated response in SCN neurons with a peak spectral sensitivity at $505 \mathrm{~nm}$ that shifts to $510 \mathrm{~nm}$ [mid-wavelength (MW) opsin] in photopic conditions, and rods appear to be required for entrainment at low light levels (Ebihara and Tsuji, 1980; Mrosovsky, 2003)

Cone (and rod) signals from the outer retina can be relayed to the SCN via pathways from melanopsin ipRGCs or nonmelanopsin RGCs (Morin et al., 2003; Sollars et al., 2003; Hattar et al., 2006). The ON-OFF responses are derived from the well known anatomical and physiological stratification of the inner plexiform layer (Wassle, 2004). ipRGCs can be monostratified with synaptic arbors in either the ON or the OFF sublamina or bistratified with arborizations in both sublamina (Pu, 1999; Provencio et al., 2002; Belenky et al., 2003; Warren et al., 2003). This is consistent with the report that ipRGCs display primarily excitatory glutamatergic, primarily inhibitory GABAergic, or both types of synaptic events (Perez-Leon et al., 2006). Transient ON-OFF responses to light are typical of ipRGCs [rodents (Berson et al., 2002; Warren et al., 2003; Perez-Leon et al., 2006); primates (Dacey et al., 2005)] and in the SCN [rat (Aggelopoulos and Meissl, 2000; Pu, 2000); mouse (Mure et al., 2007)].

In addition to the temporal properties of the transient response, the vulnerability to light adaptation confirms that this component emerges from retinal channels involving outer retinal photoreceptors. Extrinsic responses from rods and cones recorded in both rat and primate ipRGCs in vitro are eliminated under synaptic blockade or maintained light exposure without affecting the slow sustained intrinsic depolarization (Berson et al., 2002; Warren et al., 2003; Dacey et al., 2005). Bleaching adaptation by a light bright enough to photoconvert a significant fraction of the photopigment produces a prolonged desensitiza- 

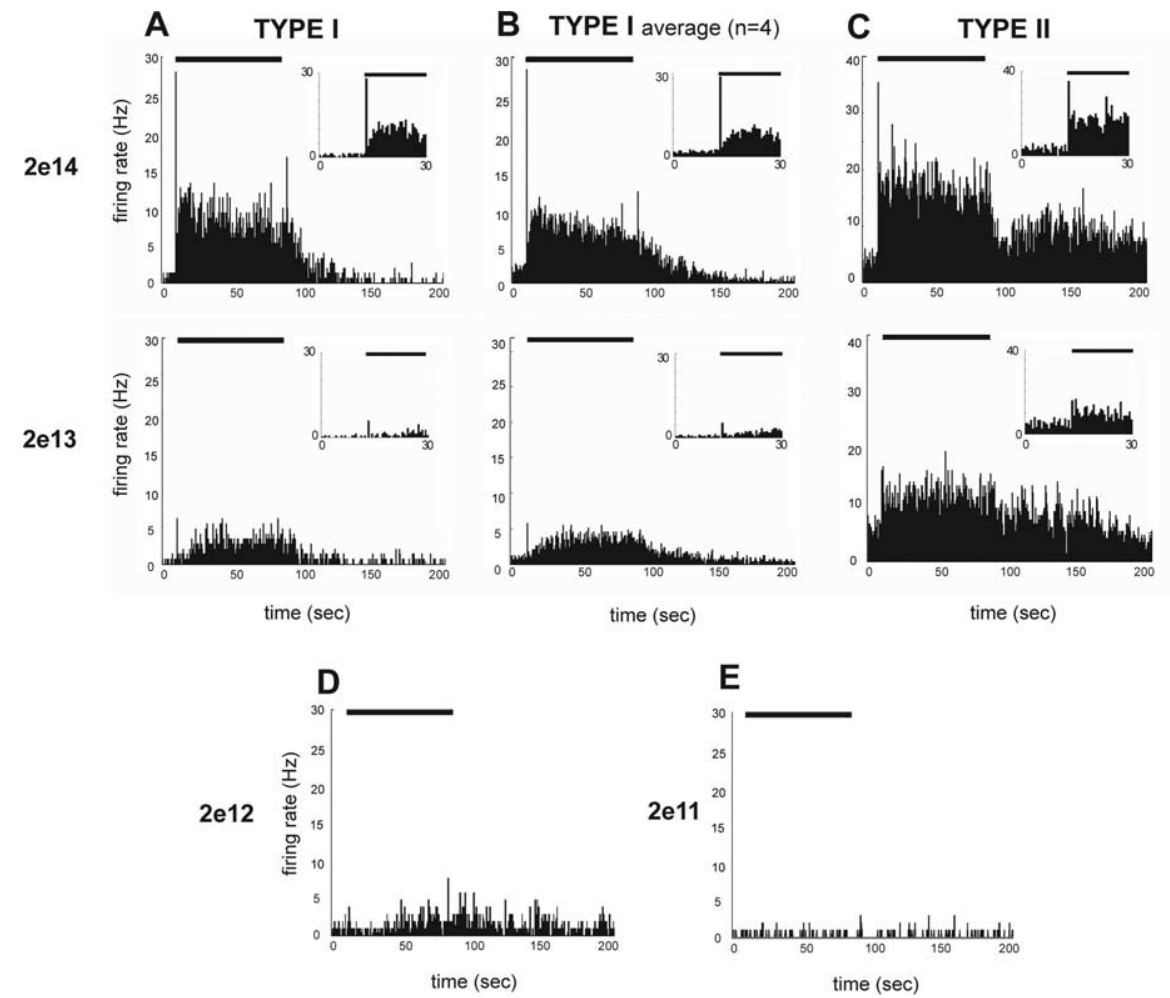

Figure 5. Single-unit responses of SCN neurons at different irradiances in the dark-adapted condition. After 30 min of dark adaptation, the animal is exposed to $500 \mathrm{~nm}$ monochromatic light at irradiances from $2 \times 10^{11}$ to $2 \times 10^{14} \mathrm{photons} / \mathrm{cm}^{2} / \mathrm{s}$ during $90 \mathrm{~s}$ (black bar). $\boldsymbol{A}, \boldsymbol{B}$, Example of a single type I neuron $(\boldsymbol{A})$ and averaged responses of type I neurons $(\boldsymbol{B})$ at $2 \times 10^{14}$ and $2 \times 10^{13}$ photons $/ \mathrm{cm}^{2} / \mathrm{s}$. C, A type ll neuronal response to light at $2 \times 10^{14}$ and $2 \times 10^{13}$ photons $/ \mathrm{cm}^{2} / \mathrm{s}$. D, E, Example of type I neuronal responses to lower irradiances at $2 \times 10^{12}$ photons $/ \mathrm{cm}^{2} / \mathrm{s}(\boldsymbol{D})$ and at $2 \times 10^{11}$ photons $/ \mathrm{cm}^{2} / \mathrm{s}(\boldsymbol{E})$ where no further light responses were detected.

tion of photoreceptors that slowly recover in the dark (Fain et al., 2001). Aggelopoulos and Meissl (2000) showed that sensitivity of the SCN neuronal response to short light pulse continuously improves with extended periods of darkness. These response dynamics may represent a cellular correlate of the effects of previous photic history on melatonin suppression by light in humans (Hebert et al., 2002; Smith et al., 2004) or phase-shifting responses in the rodent (Khammanivong and Nelson, 2000).

The sustained, slow-transient, and persistent components to the SCN derive from melanopsin photoresponses

The slow response kinetics of the sustained response to constant light are well documented features of SCN neurons dating from the early electrophysiological recordings by Groos and Mason (1978) and Meijer et al. (1986), who originally described the gradual increase of the response to light as well as the subsequent slow decay to baseline at the end of stimulation. The temporal response of ipRGCs, including the sustained response to continuous illumination, encoding of stimulus energy in the photopic range, and sluggish responses to light onsets and offsets (Berson et al., 2002; Warren et al., 2003; Dacey et al., 2005; Tu et al., 2005) are in agreement with our SCN recordings.

In addition to response kinetics, the observation that the sustained and persistent components are weakly affected by the previous bright white light exposure is consistent with melanopsin photoresponse properties. The capacity of ipRGCs to resist bleaching by light has been proposed to derive from the invertebrate-like phototransduction mechanisms of the melanopsin photopigment (Isoldi et al., 2005; Berson, 2007; Nayak et time (sec)

al., 2007). This maintenance of photic responsiveness is attributed to the bistable nature of melanopsin and its ability to use light to regenerate the chromophore as suggested in several previous in vitro (Melyan et al., 2005; Panda et al., 2005) and in vivo (Mure et al., 2007) studies.

\section{Neuronal response classes in the SCN} Sekaran et al. (2003), using calcium imaging, and, more recently, Tu et al. (2005), have described a surprising heterogeneity in the intrinsic light response properties of the adult retina, suggesting the existence of distinct melanopsin ipRGC cell classes. Tu et al. (2005) describe a type III ipRGC, characterized by a rapid onset, high light sensitivity, and very slow offset that corresponds to our type II SCN neuronal response. The authors also describe a type II ipRGC with a slow onset, lower light sensitivity, and slow offset. For decreasing irradiances, the latency increases and amplitude significantly decreases in type II ipRGCs, whereas the type III ipRGCs are relatively less affected. Based on the analogous photic response properties, it is likely that the type II melanopsin ipRGCs provide the slow-transient component we observe in type I SCN neurons, whereas the type III ipRGCs supply the sustained response typical of type II SCN neurons.

However, it appears plausible that the slow-transient and sustained components are combined in different proportions in individual SCN neurons and reflect a continuum of convergent responses originating from the different types of melanopsin ipRGCs. This convergence is consistent with previous observations that SCNprojecting RGCs have receptive fields of only $2-5^{\circ}(\mathrm{Pu}, 2000)$, whereas light-responsive SCN neurons have large receptive fields often exceeding $20^{\circ}$ in diameter (Groos and Mason, 1980).

\section{Signals from inner and outer retinal channels combine to shape the photic response of the SCN}

Our results show that SCN neurons receive a complex photic signal in which the response kinetics, response to irradiance, and resistance to bleaching are typical for channels involving both classical photoreceptors and melanopsin ipRGCs (Fig. 6). The melanopsin-based photoresponse provides the SCN with a signal that is sustained and only weakly affected by light adaptation, in line with the canonical ability of the circadian system to integrate irradiance over long time periods (Nelson and Takahashi, 1991; Dkhissi-Benyahya et al., 2007). Studies in $\mathrm{Opn}_{4}{ }^{-1-}$ mice clearly demonstrate the contribution of melanopsin in photic entrainment and light-induced phase shifts (Panda et al., 2002; Ruby et al., 2002; Hattar et al., 2003; Dkhissi-Benyahya et al., 2007). From these same studies, the retained ability of melanopsin knock-out mice to entrain and phase shift locomotor activity must necessarily rely on input from outer retinal photoreceptors.

The precise function of the transient input originating from the outer retina photoreceptors to the SCN is less clear. The transient input may compensate for the slow kinetics of the melanopsin cell response, thereby increasing the sensitivity of the cir- 
cadian system to short light pulses (Gronfier et al., 2007). This is explicitly evoked by Dacey et al. (2005) as a "priming" effect of cones to elicit the ipRGC response of melanopsin at threshold levels, in the absence of which the sustained component would not normally occur. This may alo explain the behavioral deficit observed in MW-coneless mice for duration light pulses (Dkhissi-Benyahya et al., 2007). The cone contribution could play a significant role in natural conditions where eye blinks and periodic light sampling behaviors (Boulos et al., 1996) could produce pulses of light on the retinal surface resulting in robust transient $\mathrm{ON}-\mathrm{OFF}$ responses.

Our results suggest a hierarchy of several photoreceptive streams that converge on SCN neurons (Fig. 6). A first stage of convergence combines inner retinal melanopsin ipRGCs with inputs from outer retinal cone and rod photoreceptors. Outer retinal channels carry photic information linked to rapid changes in irradiance in either photopic (cones) or scotopic (rods) domains, in relation to the spectral tuning of the involved opsins. Melanopsin ipRGCs also show heterogeneity in their responses to light (Tu et al., 2005) that appear to come together in individual SCN neurons. Several response properties still remain uncharacterized, including the response from short-wavelength cones, the contribution from rods, possible inputs from nonmelanopsin ipRGCs, and the role of melanopsin bistabilty (Mure et al., 2007). Elucidating these response properties may require devising novel strategies of light stimulation. The convergence of these photoreceptive streams, differentially encoding irradiance, wavelength, and duration, may serve several functions including extension of the irradiance operating range of the photic system, adaptation to temporal changes in light, and coding of dawn and dusk, according to the direction of change in light quality and the adaptive state of the retina.

\section{References}

Aggelopoulos NC, Meissl H (2000) Responses of neurones of the rat suprachiasmatic nucleus to retinal illumination under photopic and scotopic conditions. J Physiol (Lond) 523:211-222.

Barnard AR, Hattar S, Hankins MW, Lucas RJ (2006) Melanopsin regulates visual processing in the mouse retina. Curr Biol 16:389-395.

Baylor DA, Hodgkin AL (1974) Changes in time scale and sensitivity in turtle photoreceptors. J Physiol (Lond) 242:729-758.

Belenky MA, Smeraski CA, Provencio I, Sollars PJ, Pickard GE (2003) Melanopsin retinal ganglion cells receive bipolar and amacrine cell synapses. J Comp Neurol 460:380-393.

Berson DM (2007) Phototransduction in ganglion-cell photoreceptors. Pflügers Arch 454:849-855.

Berson DM, Dunn FA, Takao M (2002) Phototransduction by retinal ganglion cells that set the circadian clock. Science 295:1070-1073.

Bloomfield SA, Dacheux RF (2001) Rod vision: pathways and processing in the mammalian retina. Prog Retin Eye Res 20:351-384.
Boulos Z, Macchi M, Houpt TA, Terman M (1996) Photic entrainment in hamsters: effects of simulated twilights and nest box availability. J Biol Rhythms 11:216-233.

Dacey DM, Liao HW, Peterson BB, Robinson FR, Smith VC, Pokorny J, Yau KW, Gamlin PD (2005) Melanopsin-expressing ganglion cells in primate retina signal colour and irradiance and project to the LGN. Nature 433:749-754.

Dkhissi-Benyahya O, Gronfier C, De Vanssay W, Flamant F, Cooper HM (2007) Modeling the role of mid-wavelength cones in circadian responses to light. Neuron 53:677-687.

Ebihara S, Tsuji K (1980) Entrainment of the circadian activity rhythm to the light cycle: effective light intensity for a Zeitgeber in the retinal degenerate $\mathrm{C} 3 \mathrm{H}$ mouse and the normal C57BL mouse. Physiol Behav 24:523-527.

Ebling FJ (1996) The role of glutamate in the photic regulation of the suprachiasmatic nucleus. Prog Neurobiol 50:109-132.

Fain GL, Matthews HR, Cornwall MC, Koutalos Y (2001) Adaptation in vertebrate photoreceptors. Physiol Rev 81:117-151.

Freedman MS, Lucas RJ, Soni B, von Schantz M, Munoz M, David-Gray Z, Foster R (1999) Regulation of mammalian circadian behavior by nonrod, non-cone, ocular photoreceptors. Science 284:502-504.

Gingrich JA, Hen R (2000) The broken mouse: the role of development, plasticity and environment in the interpretation of phenotypic changes in knockout mice. Curr Opin Neurobiol 10:146-152. 
Gronfier C, Wright Jr KP, Kronauer RE, Czeisler CA (2007) Entrainment of the human circadian pacemaker to longer-than-24-h days, Proc Natl Acad Sci USA 104:9081-9086.

Groos G, Mason R (1978) Maintained discharge of rat suprachiasmatic neurons at different adaptation levels. Neurosci Lett 8:59-64.

Groos G, Mason R (1980) The visual properties of rat and cat suprachiasmatic neurons. J Comp Physiol [A] 135:349-356.

Hattar S, Lucas RJ, Mrosovsky N, Thompson S, Douglas RH, Hankins MW, Lem J, Biel M, Hofmann F, Foster R, Yau KW (2003) Melanopsin and rod-cone photoreceptive systems account for all major accessory visual functions in mice. Nature 424:75-81.

Hattar S, Kumar M, Park A, Tong P, Tung J, Yau KW, Berson DM (2006) Central projections of melanopsin-expressing retinal ganglion cells in the mouse. J Comp Neurol 497:326-349.

Hebert M, Martin SK, Lee C, Eastman CI (2002) The effects of prior light history on the suppression of melatonin by light in humans. J Pineal Res 33:198-203.

Isoldi MC, Rollag MD, Castrucci AM, Provencio I (2005) Rhabdomeric phototransduction initiated by the vertebrate photopigment melanopsin. Proc Natl Acad Sci USA 102:1217-1221.

Khammanivong A, Nelson DE (2000) Light pulses suppress responsiveness within the mouse photic entrainment pathway. J Biol Rhythms 15:393-405.

Knoblauch K, Shevell SK (2001) Relating cone signals to color appearance: failure of monotonicity in yellow/blue. Vis Neurosci 18:901-906.

Marc RE, Jones BW (2003) Retinal remodeling in inherited photoreceptor degenerations. Mol Neurobiol 28:139-147.

Meijer JH, Groos GA, Rusak B (1986) Luminance coding in a circadian pacemaker: the suprachiasmatic nucleus of the rat and the hamster. Brain Res 382:109-118.

Melyan Z, Tarttelin EE, Bellingham J, Lucas RJ, Hankins MW (2005) Addition of human melanopsin renders mammalian cells photoresponsive. Nature 433:741-745.

Morin LP, Blanchard JH, Provencio I (2003) Retinal ganglion cell projections to the hamster suprachiasmatic nucleus, intergeniculate leaflet, and visual midbrain: bifurcation and melanopsin immunoreactivity. J Comp Neurol 465:401-416.

Mrosovsky N (2003) Contribution of classic photoreceptors to entrainment. J Comp Physiol A Neuroethol Sens Neural Behav Physiol 189:69-73.

Mure LS, Rieux C, Hattar S, Cooper HM (2007) Restoration and enhancement of melanopsin-dependent non-visual responses by light: evidence for photopigment bistability in vivo. J Biol Rhythms 22:411-424.

Nathan J, Reh R, Ankoudinova I, Ankoudinova G, Chang B, Heckenlively J, Hurley JB (2006) Scotopic and photopic visual thresholds and spatial and temporal discrimination evaluated by behavior of mice in a water maze. Photochem Photobiol 82:1489-1494.

Nayak SK, Jegla T, Panda S (2007) Role of a novel photopigment, melanopsin, in behavioral adaptation to light. Cell Mol Life Sci 64:144-154.

Nelson DE, Takahashi JS (1991) Sensitivity and integration in a visual pathway for circadian entrainment in the hamster (Mesocricetus auratus). J Physiol (Lond) 439:115-145.

Panda S, Sato TK, Castrucci AM, Rollag MD, DeGrip WJ, Hogenesch JB,
Provencio I, Kay SA (2002) Melanopsin (Opn4) requirement for normal light-induced circadian phase shifting. Science 298:2213-2216.

Panda S, Provencio I, Tu DC, Pires SS, Rollag MD, Castrucci AM, Pletcher MT, Sato TK, Wiltshire T, Andahazy M, Kay SA, Van Gelder RN, Hogenesch JB (2003) Melanopsin is required for non-image-forming photic responses in blind mice. Science 301:525-527.

Panda S, Nayak SK, Campo B, Walker JR, Hogenesch JB, Jegla T (2005) Illumination of the melanopsin signaling pathway. Science 307:600-604

Perez-Leon JA, Warren EJ, Allen CN, Robinson DW, Lane Brown R (2006) Synaptic inputs to retinal ganglion cells that set the circadian clock. Eur J Neurosci 24:1117-1123.

Provencio I, Rollag MD, Castrucci AM (2002) Photoreceptive net in the mammalian retina. Nature 415:493.

Pu M (1999) Dendritic morphology of cat retinal ganglion cells projecting to suprachiasmatic nucleus. J Comp Neurol 414:267-274.

Pu M (2000) Physiological response properties of cat retinal ganglion cells projecting to suprachiasmatic nucleus. J Biol Rhythms 15:31-36.

$\mathrm{Pu}$ M, Xu L, Zhang H (2006) Visual response properties of retinal ganglion cells in the royal college of surgeons dystrophic rat. Invest Ophthalmol Vis Sci 47:3579-3585.

Rea MA (1998) Photic entrainment of circadian rhythms in rodents. Chronobiol Int 15:395-423.

Ruby NF, Brennan TJ, Xie X, Cao V, Franken P, Heller HC, O’Hara BF (2002) Role of melanopsin in circadian responses to light. Science 298:2211-2213.

Sakamoto K, Liu C, Tosini G (2004) Classical photoreceptors regulate melanopsin mRNA levels in the rat retina. J Neurosci 24:9693-9697.

Sekaran S, Foster R, Lucas RJ, Hankins MW (2003) Calcium imaging reveals a network of intrinsically light-sensitive inner-retinal neurons. Curr Biol Curr Biol 13:1290-1298.

Semo M, Peirson S, Lupi D, Lucas RJ, Jeffery G, Foster RG (2003) Melanopsin retinal ganglion cells and the maintenance of circadian and pupillary responses to light in aged rodless/coneless $(\mathrm{rd} / \mathrm{rd} \mathrm{cl})$ mice. Eur J Neurosci 17:1793-1801.

Smith KA, Schoen MW, Czeisler CA (2004) Adaptation of human pineal melatonin suppression by recent photic history. J Clin Endocrinol Metab 89:3610-3614.

Sollars PJ, Smeraski CA, Kaufman JD, Ogilvie MD, Provencio I, Pickard GE (2003) Melanopsin and non-melanopsin expressing retinal ganglion cells innervate the hypothalamic suprachiasmatic nucleus. Vis Neurosci 20:601-610.

Tu DC, Zhang D, Demas J, Slutsky EB, Provencio I, Holy TE, Van Gelder RN (2005) Physiologic diversity and development of intrinsically photosensitive retinal ganglion cells. Neuron 48:987-999.

Volgyi B, Deans MR, Paul DL, Bloomfield SA (2004) Convergence and segregation of the multiple rod pathways in mammalian retina. J Neurosci 24:11182-11192.

Warren E, Allen CN, Brown R, Robinson B. (2003) Intrinsic light responses of retinal ganglion cells projecting to the circadian system. Eur J Neurosci Eur J Neurosci 17:1727-1735.

Wassle H (2004) Parallel processing in the mammalian retina. Nat Rev Neurosci 5:747-757.

Wong KY, Dunn FA, Berson DM (2005) Photoreceptor adaptation in intrinsically photosensitive retinal ganglion cells. Neuron 48:1001-1010. 